\title{
SW Sex Stars, Old Novae, and the Evolution of Cataclysmic Variables
}

\author{
L. Schmidtobreick ${ }^{1}$, C. Tappert ${ }^{2}$ \\ ${ }^{1}$ European Southern Observatory, Casilla 19001, Santiago 19, Chile \\ ${ }^{2}$ Departamento de Física y Astronomía, Universidad de Valparaíso, Avda. Gran Bretaña 1112, Valparaíso, Chile
}

Corresponding author: Ischmidt@eso.org

\begin{abstract}
The population of cataclysmic variables with orbital periods right above the period gap are dominated by systems with extremely high mass transfer rates, the so-called SW Sextantis stars. On the other hand, some old novae in this period range which are expected to show high mass transfer rate instead show photometric and/or spectroscopic resemblance to low mass transfer systems like dwarf novae. We discuss them as candidates for so-called hibernating systems, CVs that changed their mass transfer behaviour due to a previously experienced nova outburst. This paper is designed to provide input for further research and discussion as the results as such are still very preliminary.
\end{abstract}

Keywords: cataclysmic variables - dwarf novae - novae - SW Sextantis stars.

\section{Introduction}

In the following we briefly review our current knowledge on the populations of old novae and SW Sextantis stars especially in the context of the evolution of cataclysmic variable stars (CVs).

\section{$1.1 \quad$ Evolution of CVs}

In general, the evolution of stable interactive binaries is driven by angular momentum loss which as such determines the mass transfer rate. The loss of angular momentum as a source for stable mass transfer implicates that the general evolutionary direction for CVs is from longer to shorter orbital periods. According to the standard model of $\mathrm{CV}$ evolution, the main source of angular momentum loss for CVs with orbital periods $P_{\text {orb }}>3 \mathrm{~h}$ is magnetic braking. Due to the continuous mass transfer, the secondary is pushed out of thermal equilibrium and becomes bloated. At a period of about $3 \mathrm{~h}$, i.e. at the upper edge of the period gap, the secondary becomes fully convective, magnetic braking ceases and only the much weaker braking through the emission of gravitational radiation continues. Therefore, the mass transfer is greatly diminished which allows the secondary star to relax into a state of thermal equilibrium and to contract to a volume corresponding to its mass. It thus loses contact with its Roche lobe and mass transfer stops completely. The angular momentum loss via gravitational radiation shrinks the orbit of the now detached and therefore hardly de- tectable binary until the secondary star fills its Roche lobe again at an orbital period of about $2 \mathrm{~h}$, i.e. at the lower edge of the gap and the binary continues as a low mass transfer $\mathrm{CV}$ below the gap. For an extensive review of the current understanding of $\mathrm{CV}$ evolution, see Knigge et al.(2011).

\section{$1.2 \quad$ SW Sex stars}

This sub-class was originally defined by Thorstensen et al. (1991). incorporating eclipsing nova-like stars with single-peaked emission lines, high velocity line wings, strong He II emission but no polarisation, and transient absorption features at orbital phases around $\phi=0.5$. The radial velocity curves show an offset of 0.2 cycle with respect to the phase defined by the eclipse. Today, SW Sex stars are considered novalike stars with an extremely high mass transfer rate (see e.g. Rodríguez-Gil et al. 2007a,b). This is supported by the high temperatures found for the white dwarfs in these systems (Townsley \& Gänsicke, 2009). While SW Sex stars used to be considered as rare and strange objects with unusual behaviour, it has been shown in the last years that they in fact represent the dominant $\mathrm{CV}$ population in the orbital period range between about three and four hours (Schmidtobreick et al., 2012). From a sample of CVs that was purely seleceted to have an orbital period between three and four hours, they find that the percentage of SW Sex stars in this range must exceed $85 \%$. According to the standard model, all long period CVs have to evolve through this period range before entering the gap and will thus share the SW Sex characteristics 
during that time. This makes the SW Sex phenomenon a phase in the secular evolution of CVs (Schmidtobreick et al. in preparation).

\subsection{Old novae}

Classical novae are CVs that experience a thermonuclear runaway on the surface of the white dwarf where the accreted material has reached a critical mass. In this process, the binary is not destroyed and mass transfer is usually re-established within a few months. It is therefore safe to assume that nova explosions are recurrent events and part of the evolution of every CV with a sufficiently high mass transfer rate to accumulate the necessary material on the white dwarf surface within its lifetime. Inbetween the nova events, the behaviour of the CV depends on properties like orbital period, mass-transfer rate, and magnetic field of the white dwarf that also determine its subtype. In addition, the hibernation model predicts changes of the mass transfer rate in the evolution of the pre- and postnova: after an initial phase of high mass transfer rate due to the secondary star being driven strongly out of thermal equilibrium via irradiation from the eruptionheated white dwarf - which increases the distance between the two stars, the binary should descend into a long state of low mass transfer once the white dwarf cooled down and the secondary is allowed to relax more towards thermal equilibrium. (Shara et al., 1986; Prialnik \& Shara, 1986). Some potential evidence for hibernation has been presented in the form of old nova shells around CVs that have previously been known as low mass transfer systems, i.e. Z Cam (Shara et al., 2007) and AT Cnc (Shara et al., 2012). However this interpretation is not exclusive, as the presence of nova shells around dwarf novae could also indicate that all types of CV (including dwarf novae) can experience a nova explosion during their lifetime without necessarily undergoing cyclic changes of the mass transfer rate.

\section{What Can We Learn Combining Our Knowledge from Old Novae and SW Sex Stars?}

Several years ago, we conducted a project investigating old novae which had experienced large outburst amplitudes (Schmidtobreick et al., 2003; 2005). The idea behind this was that since the absolute magnitude of a nova explosion depends mainly on the mass of the white dwarf (Livio, 1992) it thus differs only slightly for different systems. Thus, novae with large outburst amplitudes are likely to be intrinsically faint CVs and therefore candidates for low mass transfer rate systems.
Two systems of our sample, V842 Cen and XX Tau, show spectroscopic properties that, compared to other old novae, indicate a rather low mass transfer rate. The spectra present comparatively strong Balmer emission lines from $\mathrm{H} \alpha$ bluewards down to H11. A weak HeII emission line is present at $469 \mathrm{~nm}$ and indicates a hot component in the system, but at the same time the presence and strength of the HeI series is evidence for a significant amount of cooler material. We note that most old novae do not share these characteristics (e.g., Ringwald et al. 1996, Tappert et al. 2012, 2014). For XX Tau, preliminary analysis of long-term photometric monitoring covering a range of $150 \mathrm{~d}$ furthermore shows a probable dwarf-nova like outburst with an amplitude of $\sim 0.8$ mag and a duration of $\sim 10 \mathrm{~d}$ (Tappert, private communication), which represents additional evidence for a comparatively low mass transfer rate.

The approach by Tappert et al. (2012) to re-discover lost old novae revealed two more such candidates from spectroscopy: V2109 Oph and V728 Sco. In particular V728 Sco which has also been observed photometrically can be considered a low mass transfer system as it also shows frequent stunted dwarf novae outbursts (Tappert et al., 2013a).

For three of these low mass transfer old novae, i.e. V842 Cen, V728 Sco, and XX Tau, at least rough values for the orbital period could be established (Woudt et al., 2009; Luna et al., 2012; Tappert et al., 2013a; Rodríguez-Gil \& Torres, 2005) and they all fall into the range of SW Sex stars, i.e. between 3 and 4 hours.

On the one hand, this period range is thus dominated by high mass transfer CVs. On the other hand, also the majority of old novae have high mass transfer rates. In fact, the period distribution of old novae shows a distinctive peak in the $2.8-5 \mathrm{~h}$ period range, while few novae are found in the period bins that are dominated by dwarf novae (e.g., Tappert et al. 2013b, Schmidtobreick \& Tappert 2014). Thus, the indicated low mass transfer nature of V842 Cen, V728 Sco and $\mathrm{XX}$ Tau is somewhat surprising.

For the sake of completeness, we point out that other low mass transfer novae are known. For example, the system V446 Her (Porb $=4.97 \mathrm{~h}$, Thorstensen \& Talor, 2000) shows frequent "stunted" dwarf-nova like outbursts (Honeycutt et al. 1998). However, we here restrict our discussion to systems with orbital periods in the $3-4 \mathrm{~h}$ range.

When thinking about a reason why several old novae would appear as low mass transfer systems even though some of them are even situated in the SW Sex regime, hibernation comes naturally to mind. It seems an attractive explanation that these CVs have indeed been SW Sex stars which due to the nova explosion in the recent past were pushed into a low state and thus experience low mass transfer rates at the moment. In 
this context we point out that V728 Sco in outburst presents a triangular eclipse shape that is typical for SW Sex stars (Tappert et al., this volume).

\section{Conclusions and Future Work}

CVs with orbital periods between 3 and 4 hours are in general SW Sex stars and as such experience high masstransfer rates. However, we find evidence for three old novae with orbital periods in this range that should be of SW Sex type but are instead low mass transfer systems. We tentatively conclude that the mass transfer of these systems was changed by the nova eruption, similar to what is proposed in the hibernation scenario. Whether the mass transfer in these systems will completely cease or just remain on a low level before rising up again remains to be investigated. To better understand the recent mass transfer history and to thus shed light on the evolutionary state of these systems, it would be important to measure the dynamical masses and the temperatures of the two components in order to determine the binary solution. For this, it is valuable to have a system like V728 Sco in the sample which as eclipsing binary offers more opportunities to determine the binary parameters.

To test the idea that the low mass transfer novae in the SW Sex regime are affected by hibernation, it will be essential to check for the few existing dwarf novae in the SW Sex range whether they have experienced a nova eruption in the past. We thus started an observational project to look for nova shells around these objects. Other possible candidates for hibernating CV could be found among the so-called pre-CVs, white-dwarf/mainsequence binary which are detached systems. Examples for such candidates are LTT 560 (Tappert et al., 2011) and QS Vir (Drake et al., 2014, and references therein) which both have orbital periods in the SW Sex range and show evidence of accretion. If indeed a significant number of these low mass transfer systems in the SW Sex regime can be shown to have experienced a nova outburst in the past, this would not only be strong evidence for the hibernation scenario but at the same time also prove the evolutionary significance of the SW Sex stars as it would yield a natural explanation for the few remaining CVs in the $3-4 \mathrm{~h}$ period range that do not follow the SW Sex behaviour.

\section{Acknowledgement}

This research was supported by FONDECYT Regular grant 1120338 (CT). We gratefully acknowledge the use of the SIMBAD database, operated at CDS, Stras- bourg, France, and of NASA's Astrophysics Data System Bibliographic Services. We thank an anonymous referee for valuable comments.

\section{References}

[1] Knigge C., Baraffe I., Patterson J., 2011, APJS 194, 28 doi:10.1088/0067-0049/194/2/28

[2] Drake J. J., Garraffo C., Takei D, Gänsicke B., 2014, MNRAS 437, 3842

doi:10.1093/mnras/stt2186

[3] Honeycutt R. K., Robertson J. W., Turner G. W., Henden A. A., 1998, ApJ 495, 933 doi:10.1086/305299

[4] Livio, M. 1992, in Vina del Mar Workshop on Cataclysmic Variable Stars, edited by N. Vogt, ASP Conf. Ser. 29, 4.

[5] Luna G. J. M., Diaz M. P., Brickhouse N. S., Moraes M., 2012, MNRAS, 423, L75. doi:10.1111/j.1745-3933.2012.01260.x

[6] Prialnik, D., \& Shara, M. M. 1986, ApJ, 311, 172. doi:10.1086/164763

[7] Ringwald F. A., Naylor T., Mukai K., 1996, MNRAS 281, 192 doi:10.1093/mnras/281.1.192

[8] Rodríguez-Gil P., Schmidtobreick L., Gänsicke B. T., 2007a, MNRAS 374, 1359 doi:10.1111/j.1365-2966.2006.11245.x

[9] Rodríguez-Gil P., Gänsicke B. T., Hagen H.-J. et al., 2007b, MNRAS 377, 1747 doi:10.1111/j.1365-2966.2007.11743.x

[10] Rodríguez-Gil P., Torres M. A. P., 2005, A\&A, 431, 289

[11] Schmidtobreick L., Tappert C., Saviane I., 2003a, MNRAS 342, 145 doi:10.1046/j.1365-8711.2003.06523.x

[12] Schmidtobreick L., Tappert C., Bianchini A., Mennickent R. E. 2003b, A\&A, 410, 943.

[13] Schmidtobreick L., Tappert C., Bianchini A., Mennickent R. E., 2005, A\&A, 432, 199.

[14] Schmidtobreick L., Rodríguez-Gil P., Gänsicke B. T., 2012, Mem. S.A.It. Vol. 83, 610

[15] Schmidtobreick L., Tappert C., 2014, Stella Novae: Future and Past Decades. Woudt \& Ribeiro (eds), ASP in press

[16] Shara, M. M., Livio, M., Moffat, A. F. J., \& Orio, M. 1986, ApJ 311, 163 
[17] Shara M. M., Martin C. D., Seibert M., Rich R. M., Salim S., Reitzel D., Schiminovich D., Deliyannis C. P., Sarrazine A. R., Kulkarni S. R., Ofek E. O., Brosch N., Lépine S., Zurek D., De Marco O., Jacoby G., 2007, Nature 446, 159

[18] Shara M. M., Mizusawa T., Wehinger P., Zurek D., Martin C. D., Neill J. D., Forster K., Seibert M., 2012, ApJ 758, 121.

[19] Tappert C., Gänsicke B. T., Schmidtobreick L., Ribeiro T., 2011, A\&A 532, 129

[20] Tappert C., Ederoclite A., Mennickent R. E., Schmidtobreick L., Vogt N. 2012, MNRAS, 423, 2476 .

[21] Tappert C., Vogt N., Schmidtobreick L., Ederoclite A., Vanderbeke J., 2013a, MNRAS 431, 92

[22] Tappert C., Schmidtobreick L., Vogt N., Ederoclite, A., 2013b, MNRAS 436, 2412

[23] Tappert C., Vogt N., Della Valle M., Schmidtobreick L., Ederoclite A., 2014, MNRAS in press
[24] Thorstensen J. R., Taylor C. J., 2000, MNRAS 312,629

[25] Thorstensen J. R., Ringwald F. A., Wade R. A., Schmidt G. D., Norsworthy J. E., 1991, AJ 102, 272

[26] Townsley D. M., Gänsicke B. T., 2009, ApJ 693, 1007

[27] Woudt, P. A., Warner, B., Osborne, J., Page, K. 2009, MNRAS, 395, 2177.

\section{DISCUSSION}

VITALY NEUSTROEV: You claim that some old novae have a very small mass transfer rate. Does it mean that these stars should show DN-type outbursts?

LINDA SCHMIDTOBREICK: Yes, indeed. And some stars do show such outbursts. We don't yet have a lot of data but we have observed several outbursts for V728 Sco. 